\title{
Characterizing Hyperbolic Functions over a Hyperplane ${ }^{\dagger}$
}

\section{Kanet PONPETCH*}

27 Moo 2, Khlong Yai Sub-district, Tamod District, Phatthalung 93160 Thailand

(*Corresponding author's e-mail: kanet.bkp@gmail.com)

Received: 30 November 2018, Revised: 20 February 2019, Accepted: 28 February 2019

\begin{abstract}
A brief account of our recent works dealing with the problem of characterizing hyperbolic functions through the use of functional equations with side conditions is reported. Specifically, the fact that each of the $n$ functions having a zero sum over $n$ points lying in a hyperplane is necessarily a linear function, is generalized and the results so obtained are applied to characterize hyperbolic functions.
\end{abstract}

Keywords: Characterizing, hyperplane, hyperbolic function

Mathematics Subject Classification: 39B22, 33B10

\section{Introduction}

As seen from [8, Chapter 3] and [7, Chapter 13], the investigation about using functional equations to characterize trigonometric-hyperbolic functions have long been of interest. We report here our recent works which were originated from the 2004 work of Benz [2], who confirmed the result of Davison [1] by showing that the functional equation $f(x) f(y) f(z)=f(x)+f(y)+f(z)$, with the three angles $x, y, z$ of a non-degenerate triangle, can be used to characterize the tangent function. In 2014, Hengkrawit et al. [3] generalized this result by solving a functional equation, with $n$ parameters representing the angles of a non-degenerate convex $n$-gon, and used it to characterizes the tangent function. In 2016, Hengkrawit et al. [4] solved another functional equation, different from [3], and used it to characterize the tangent function. Adopting the method and techniques extracted from the proof in [3], we obtain in [6]:

Theorem 1. Let $n$ be an integer $\geq 3$, and let I denote the closed interval $[a, b]$ with $b>a$. Then the functions $\phi_{i}: I \rightarrow \mathbb{R}(i=1,2, \ldots, n)$ satisfy the constant sum functional equation

$\sum_{i=1}^{n} \phi_{i}\left(x_{i}\right)=T_{1}, \quad x_{i} \in I(i=1,2, \ldots, n)$,

subject to the hyperplane condition

$\sum_{i=1}^{n} x_{i}=T_{2}$

where $T_{1}, T_{2}$ are real constants with

$\frac{n(2 a+b)}{3}<T_{2}<\frac{n(a+2 b)}{3}$,

if and only if, there exists an additive function $A: \mathbb{R} \rightarrow \mathbb{R}$ such that

$\phi_{i}(x)=A(x)-A\left(T_{2} / n\right)+\gamma_{i} \quad(i=1,2, \ldots, n)$,

Presented at the International Conference in Number Theory and Applications 2018: December $13^{\text {th }}-15^{\text {th }}, 2018$ 
where the constants $\gamma_{i}$ satisfy

$\sum_{i=1}^{n} \gamma_{i}=T_{1}$

The functional equation in the above theorem deals with functions with constant sum over points lying in a hyperplane, and its application to characterize the hyperbolic functions is outlined in the next section.

\section{Hyperbolic functions}

The first step towards characterization is to derive additive formulas for hyperbolic functions which is given in the next lemma.

Lemma 2. I. Let $n$ be an integer $\geq 2$. If $y_{1}, \ldots, y_{n} \in \mathbb{R}$, then

$\sinh \left(y_{1}+\cdots+y_{n}\right)=\sum_{M=0}^{\left\lfloor\frac{n-1}{2}\right\rfloor} \sum_{1 \leq i_{1}<\cdots<i_{2 M+1} \leq n} \mathscr{S}_{n}\left(i_{1}, \ldots, i_{2 M+1}\right)$,

where

$\mathscr{S}_{n}\left(i_{1}, \ldots, i_{2 M+1}\right):=\left(\prod_{k=1}^{2 M+1} \frac{\sinh y_{i_{k}}}{\cosh y_{i_{k}}}\right)\left(\prod_{j=1}^{n} \cosh y_{j}\right)$

and

$\cosh \left(y_{1}+\cdots+y_{n}\right)=\sum_{M=0}^{\left\lfloor\frac{n}{2}\right\rfloor} \sum_{1 \leq i_{1}<\cdots<i_{2 M} \leq n} \mathscr{C}_{n}\left(i_{1}, \ldots, i_{2 M}\right)$,

where $\mathscr{C}_{n}\left(i_{1}, \ldots, i_{2 M}\right):= \begin{cases}\left(\prod_{k=1}^{2 M} \frac{\sinh y_{i_{k}}}{\cosh y_{i_{k}}}\right)\left(\prod_{j=1}^{n} \cosh y_{j}\right) & \text { if } M \neq 0 \\ \prod_{j=1}^{n} \cosh y_{j} & \text { if } M=0 .\end{cases}$

II. Let $n \in \mathbb{N}, n \geq 3$, let $A_{1}, \ldots, A_{n-1} \in \mathbb{R}$ and let

$h_{1}(n):=\sum_{M=1}^{\left\lfloor\frac{n-1}{2}\right\rfloor} \sum_{1 \leq i_{1}<i_{2}<\cdots<i_{2 M} \leq n-1} \prod_{k=1}^{2 M} \tanh A_{i_{k}}$

$h_{2}(n):=\sum_{M=0}^{\left\lfloor\frac{n-2}{2}\right\rfloor} \sum_{1 \leq i_{1}<i_{2}<\cdots<i_{2}} \prod_{k=1}^{2 M+1} \tanh A_{i_{k}}$.

If $1+h_{1}(n) \neq 0$, then

$\tanh \left(A_{1}+\cdots+A_{n-1}\right)=\frac{h_{2}(n)}{1+h_{1}(n)}$.

Strategically combining Theorem 1 with Lemma 2, we arrive at the following characterizations of the hyperbolic sine, cosine and tangent functions. 
Theorem 3. Let $n$ be an integer $\geq 3$.

I. Let $b>a$, the functions $f_{j}:[a, b] \rightarrow \mathbb{R}$ and $g_{j}:[a, b] \rightarrow[1, \infty)(j=1, \ldots, n)$ satisfying

$\sum_{M=0}^{\left\lfloor\frac{n}{2}\right\rfloor} \sum_{1 \leq i_{1}<\cdots<i_{2 M} \leq n} \mathfrak{C}_{n}\left(f_{j}, g_{j} ; i_{1}, \ldots, i_{2 M}\right)=1$,

where $\mathfrak{C}_{n}\left(f_{j}, g_{j} ; i_{1}, \ldots, i_{2 M}\right):= \begin{cases}\left(\prod_{k=1}^{2 M} \frac{f_{i_{k}}\left(x_{i_{k}}\right)}{g_{i_{k}}\left(x_{i_{k}}\right)}\right)\left(\prod_{j=1}^{n} g_{j}\left(x_{j}\right)\right) & \text { if } M \neq 0 \\ \prod_{j=1}^{n} g_{j}\left(x_{j}\right) & \text { if } M=0,\end{cases}$

subject to the condition

$$
\begin{aligned}
\sinh ^{-1} \circ f_{j} & =\cosh ^{-1} \circ g_{j} \quad(j=1, \ldots, n) \\
\sum_{j=1}^{n} x_{j} & =L_{1},
\end{aligned}
$$

where $L_{1}$ is a constant belonging to the range $\frac{n(2 a+b)}{3}<L_{1}<\frac{n(a+2 b)}{3}$, are given by $f_{j}(x)=\sinh \left(A_{1}(x)-A_{1}\left(L_{1} / n\right)+d_{j}\right), g_{j}(x)=\cosh \left(A_{1}(x)-A_{1}\left(L_{1} / n\right)+d_{j}\right)$, where $A_{1}$ is an additive function on $\mathbb{R}$ and the constants $d_{j}$ satisfy $\sum_{j=1}^{n} d_{j}=0$. II. Let $b>a$. The functions $f_{j}:[a, b] \rightarrow \mathbb{R}$ and $g_{j}:[a, b] \rightarrow[1, \infty)(j=1, \ldots, n)$ satisfying

$\sum_{M=0}^{\left\lfloor\frac{n-1}{2}\right\rfloor} \sum_{1 \leq i_{1}<\cdots<i_{2 M+1} \leq n} \mathfrak{S}_{n}\left(f_{j}, g_{j} ; i_{1}, \ldots, i_{2 M+1}\right)=0$

where $\mathfrak{S}_{n}\left(f_{j}, g_{j} ; i_{1}, \ldots, i_{2 M+1}\right):=\left(\prod_{k=1}^{2 M+1} \frac{f_{i_{k}}\left(x_{i_{k}}\right)}{g_{i_{k}}\left(x_{i_{k}}\right)}\right)\left(\prod_{j=1}^{n} g_{j}\left(x_{j}\right)\right)$, subject to the condition

$$
\begin{aligned}
\sinh ^{-1} \circ f_{j} & =\cosh ^{-1} \circ g_{j} \quad(j=1, \ldots, n) \\
\sum_{j=1}^{n} x_{j} & =L_{2},
\end{aligned}
$$

where $L_{2}$ is a constant belonging to the range $\frac{n(2 a+b)}{3}<L_{2}<\frac{n(a+2 b)}{3}$, are given by $f_{j}(x)=\sinh \left(A_{2}(x)-A_{2}\left(L_{2} / n\right)+\ell_{j}\right), g_{j}(x)=\cosh \left(A_{2}(x)-A_{2}\left(L_{2} / n\right)+d_{j}\right)$, where $A_{2}$ is an additive function on $\mathbb{R}$ and the constants $\ell_{i}$ satisfy $\sum_{j=1}^{n} \ell_{j}=0$. III. Let $b>a$. The functions $f_{j}:[a, b] \rightarrow(-1,1)(j=1, \ldots, n)$ satisfying

$$
\sum_{j=1}^{n} f_{j}\left(x_{j}\right)=-\sum_{M=1}^{\left\lfloor\frac{n-1}{2}\right\rfloor} \sum_{1 \leq i_{1}<\cdots<i_{2}} \prod_{k=1}^{2 M+1} f_{i_{k}}\left(x_{i_{k}}\right), \quad x_{j} \in I(j=1, \ldots, n),
$$

subject to the two conditions

$$
\begin{aligned}
& \sum_{j=1}^{n} x_{i}=L_{3}, \\
& 1+\sum_{M=1}^{\left\lfloor\frac{n-1}{2}\right\rfloor} \sum_{1 \leq i_{1}<\cdots<i_{2} \leq n-1} \prod_{k=1}^{2 M} f_{i_{k}}\left(x_{i_{k}}\right) \neq 0,
\end{aligned}
$$


where $L_{3}$ is a constant belonging to the range $\frac{n(2 a+b)}{3}<L_{3}<\frac{n(a+2 b)}{3}$, are given by

$f_{j}(x)=\tanh \left(A_{3}(x)-A_{3}(L / n)+t_{j}\right) \quad(j=1, \ldots, n)$,

where $A_{3}$ is an additive function on $\mathbb{R}$, and the constants $t_{j}$ satisfy $\sum_{j=1}^{n} t_{j}=0$.

In passing, let us remark that characterizations along the same vein as above for the trigonometric sine and cosine functions have already been carried out in [5].

\section{References}

[1] T Davison. Report of Meeting: The Fortieth International Symposium on Functional Equations. Aequationes Math. 2003; 65, 292.

[2] W Benz. The functional equation $f(x) f(y) f(z)=f(x)+f(y)+f(z)$. Aequationes Math. 2004; 68, $117-20$.

[3] C Hengkrawit, V Laohakosol and K Ponpetch, Functional equations characterizing the tangent function over a convex polygon. Aequationes Math. 2014; 88, 201-10.

[4] C Hengkrawit and K Ponpetch, Functional equations characterizing the tangent function over a convex polygon II. Thai J. Sci. Technol.. 2016; 5, 113-8.

[5] K Ponpetch, S Mavecha and V Laohakosol. Functions with constant sums over a hyperplane and applications. Publications de I' Institut Mathematique. 2019; 105(119), 65-80.

[6] K Ponpetch, V Laohakosol and S Mavecha. Functional equations characterizing the sine and cosine function over a convex polygon. Appl. Math. E-Notes, in press.

[7] J Aczél and J Dhombres. Functional Equations in Several Variables. Cambridge University Press, Cambridge, 1989.

[8] Pl Kannappan. Functional Equations and Inequalities with Applications. Springer, Heidelberg, 2009. 